\title{
INFLORESCENCE DEFICIENT IN ABSCISSION-like is an abscission- associated and phytohormone-regulated gene in flower separation of Lupinus luteus
}

\author{
Emilia Wilmowicz ${ }^{1} \cdot$ Agata Kućko $^{1}$ [D $\cdot$ Maciej Ostrowski ${ }^{2} \cdot$ Katarzyna Panek $^{1}$
}

Received: 1 December 2017 / Accepted: 1 February 2018 / Published online: 5 February 2018

(c) The Author(s) 2018. This article is an open access publication

\begin{abstract}
Abscission is a natural process that occurs to facilitate shedding of no longer needed organs, but on the other hand, can be triggered by certain environmental conditions, e.g. biotic or abiotic stresses. Regardless of the stimuli, organ shedding takes place specifically at the abscission zone (AZ). A signaling pathway that controls this process in Arabidopsis thaliana from ligand to receptors has been proposed. However, knowledge concerning the influence of plant hormones on these molecular elements still remains enigmatic. Excessive and premature flower abscission in the crop species Lupinus luteus L. is a process of substantial interest to the agricultural industry, as it can affect yield. Our strategy combined molecular studies, comprehensive ultrastructural and histological analysis, as well as exogenous hormone treatment to describe the contribution of the Lupinus IDA-like gene in flower abscission. In the AZ of the naturally abscised flowers, the differentiation of morphologically distinct cells characterized by progressive degradation processes was accompanied by LIIDL mRNA accumulation. A similar effect was observed following early steps of AZ activation and after abscisic acid or ethylene treatments. These phytohormones, previously pointed out as key stimulators of flower separation, altered the temporal expression pattern of LIIDL. Exogenous EPIP peptide synthesized on the basis of LIIDL sequence, significantly increased flower abortion rate, which indicates that this motif governs protein activity. In conclusion, our data provide new evidence for LIIDA involvement in both the early and late events of flower abscission supported by detailed spatiotemporal characterization of AZ cell structure and ultrastructure.
\end{abstract}

Keywords Abscisic acid · Abscission zone · Ethylene · INFLORESCENCE DEFICIENT IN ABSCISSION-like · Lupinus luteus $\cdot$ Cell ultrastructure

\section{Abbreviations}

ABA Abscisic acid

AZ Abscission zone

ET Ethylene
LIIDL INFLORESCENCE DEFICIENT IN ABSCISSION-like

RT-qPCR Reverse transcription-quantitative polymerase chain reaction
Electronic supplementary material The online version of this article (https://doi.org/10.1007/s10725-018-0375-7) contains supplementary material, which is available to authorized users.

Agata Kućko

kuckoa@poczta.onet.pl

1 Chair of Plant Physiology and Biotechnology, Nicolaus Copernicus University, 1 Lwowska Street, 87-100 Torun, Poland

2 Department of Biochemistry, Nicolaus Copernicus University, 1 Lwowska Street, 87-100 Toruń, Poland

\section{Introduction}

The shedding of various plant organs is a part of a natural developmental program. This phenomenon is often associated with reproduction, senescence, defense and response on stress factors. However, from the agronomic point of view, excessive separation of generative organs e.g. in citrus, tomato, olive, pepper, apple and legumes, leads to heavy economic loss and affects benefits of cultivators (Agustí et al. 2007; Aloni et al. 1996; Botton et al. 2011; Couzigou et al. 2016; Gomez-Jimenez et al. 2010; Meir et al. 2010; Wilmowicz et al. 2016). It has been experimentally 
established that flowers and fruits abscission occurs after activation of a functionally specialized group of cells called abscission zone (AZ). This zone is located usually at their bases (Estornell et al. 2013). Investigations of endogenous factors implicated in organ separation are likely to provide basic information on functional significance of such biomolecules. The signals thought to govern abscission are hormonal in nature. Among them, ethylene (ET), abscisic acid (ABA) and jasmonates are significant abscission accelerators, whereas auxins, gibberellins and brassinosteroids prevent that process (Estornell et al. 2013; Wilmowicz et al. 2016). ET is considered as an effector of changes leading to organ separation. The action of ET includes stimulation of enzymes involved in cell wall remodeling and middle lamellae dissolution, such as peroxidases, polygalacturonases, expansins, as well as esterases (Meir et al. 2010).

During the last decade, proteins critical for organ abscission, e.g. INFLORESCENCE DEFICIENT IN ABSCISSION (IDA) - ligand for leucine-rich repeat receptor-like kinases HAESA/HAESA-LIKE2 (HAE/HSL2), as well as other regulatory components, like EVERSHED, NEVERSHED and CAST AWAY, have been identified in Arabidopsis thaliana (Burr et al. 2011; Butenko et al. 2003; Cho et al. 2008; Liu et al. 2013). IDA and IDA-like (IDL) genes, encoding small secreted proteins, determine organ shedding e.g. in Arabidopsis thaliana, Litchi chinensis, as well as citrus (Estornell et al. 2015; Stenvik et al. 2008; Ying et al. 2016). These proteins contain an a conserved EPIP motifs (Stenvik et al. 2008). The ida mutant fails to undergo floral organ separation, whereas overexpression of IDA leads to premature abscission (Butenko et al. 2003; Stenvik et al. 2006). It was experimentally established that IDA-HAE/HSL2 complex induces a MITOGEN-ACTIVATED PROTEIN KINASE (MAP) cascade within the cytoplasm of AZ cells. This pathway suppresses KNOTTED-LIKE HOMEOBOXlike (KNOX-like) transcription factor-KNAT1, which in turn allows KNAT2 and KNAT6 to induce organ abscission (Cho et al. 2008; Shi et al. 2011).

Yellow lupine (Lupinus luteus L.) has a number of economic, pro-ecological and agronomical advantages. However, unexpected and premature abscission of lupine flowers is a major limiting factor for productivity. This process concerns $40-90 \%$ flowers per plant, which consequently leads to yield decrease (Prusiński and Borowska 2001). On the other hand, it makes an excellent model to study separation phenomenon. Our previous histological analysis showed that the flower AZ is located at the base of this organ (Frankowski et al. 2015a). Formation, as well as early steps of AZ activation, were correlated with changes in the mRNA level of $B L A D E-O N-P E T I O L E$ gene (LIBOP). The LIBOP is additionally upregulated by phytohormonal stimulators of flower abscission, e.g. ABA and ET (Frankowski et al. 2015a). We revealed also, that the artificial activation of AZ by flower removal caused accumulation of ABA in AZ cells. However, the effect of ABA on flower abortion resulted from ABA's stimulatory role in the ET biosynthesis pathway (Wilmowicz et al. 2016).

It was proposed that IDA functions in ET-response pathway in L. chinensis, Populus and palm oil (Ying et al. 2016). Conversely, IDA from A. thaliana has been postulated to be regulated in an ET-independent manner (Butenko et al. 2003). It seems that the elements of the genetic pathway that leads to organ separation are similar. Nevertheless, their activity is controlled in particular plant species by various plant hormones and other factors. This phenomenon can be a result of evolutionary adaptation to different lifestyles and environments. Therefore, understanding the molecular regulation of abscission, especially in crop species, is extremely relevant for production control. All results are of paramount importance not only for researchers but especially for breeders working in this field. The knowledge helps to elucidate abscission and allows characterization and selection of new cultivars/species with an improved characteristic.

Here, we performed histological and ultrastructural analyses of flower $\mathrm{AZ}$ in order to select proper tissue fragments for analyses. We also determined the cytological and morphological characteristics associated with abscission process. Furthermore, we present a report of identified LIIDL gene in L. luteus. This gene's transcriptional activity during early and late stages of separation events, as well as after treatments with phytohormonal modulators of abscission was investigated. On the basis of physiological studies, the functional contribution of EPIP motif from LIIDA was tested. Our paper is a new evidence on the phytohormonedependent regulation of $L I I D L$ gene during flower separation processes in yellow lupine at the level of $\mathrm{AZ}$ cells.

\section{Materials and methods}

\section{Plant material}

The L. luteus L. epigonal cv. Taper was used in the study. The plants were cultivated under the conditions described by Frankowski et al. (2015b). AZs were collected from the pedicels fragments of fully opened non-abscised (Inactive AZ, Supplementary Fig. 1a) and naturally abscised (Natural Active AZ, Supplementary Fig. 1b) flowers. We excised section $1 \mathrm{~mm}$ above and $1 \mathrm{~mm}$ below flower bases. In order to artificially activate $\mathrm{AZ}$, individual flowers were removed from plants by sharp razor blade above the AZ (Artificially Activated AZ, Supplementary Fig. 1c, d) and the tissue fragments containing AZs were collected after 2, 4, 6, 8, 16 and $24 \mathrm{~h}$. In parallel, AZs from non-abscised flowers (Supplementary Fig. 1a) were harvested at the same time variants (Control AZ). 
We also examined the influence of plant hormones on LIIDL transcriptional activity. Inactive AZs were treated with solutions of $0.1 \mathrm{mM}$ abscisic acid $( \pm \mathrm{ABA})$ or $0.01 \mathrm{mM}$ nordihydroguaiaretic acid (NDGA - an inhibitor of ABA biosynthesis) prepared in $0.05 \%$ Tween 20 . Ethylene $(100 \mu \mathrm{L} / \mathrm{L})$ or inhibitor of its action (NBD-2,5-norbornadiene, $1 \mu \mathrm{L} / \mathrm{L}$ ), were put via a syringe with septum into the glass containers with enclosed plants. At least 15 plants were used in each treatment and all experiments were replicated three times ( 3 pots $\times 5$ plants $\times 3$ independent repeats $=45$ plants). AZs sections for expression analyses were immediately frozen in liquid nitrogen and stored at $-80^{\circ} \mathrm{C}$.

\section{Amplification and cloning of LIIDL CDNA}

Total RNA for identification of LlIDL was isolated from tissue fragments containing AZ dissected from naturally abscised flowers (Natural Active AZ, Supplementary Fig. 1b). We used the NucleoSpin RNA Plant kit, MACHEREY-NAGEL GmbH \& Co. KG (Düren, Germany) according to the manufacturer's recommendations. One $\mu \mathrm{g}$ of total RNA primed with anchored oligo $(\mathrm{dT})_{18}$ was used for first strand synthesis with Transcriptor First Strand cDNA Synthesis Kit (ROCHE Diagnostics GmbH, Germany). PCR reactions with degenerated primers (Supplementary Table 1) were performed in the T3 Thermocycler (Biometra, Göttingen, Germany). Reaction's condition described previously by Frankowski et al. (2015b) were applied. Amplified cDNA fragments were isolated from an agarose gel with the GeneMATRIX Agarose Out DNA Purification Kit (EurX, Gdańsk, Poland) and cloned using the Strata Clone PCR Cloning Kit (Agilent Technologies, Santa Clara, USA) according to manufacturer's recommendations. Plasmid DNA was purified with the GeneMATRIX PLASMID MINIPREP DNA Purification Kit (EurX, Gdańsk, Poland) and sequenced by "Genomed S.A." (Warsaw, Poland). A full-length cDNA for $L I I D L$ was obtained using the BD SMART RACE cDNA Amplification Kit (Clontech-Takara Bio Europe, Saint-Germain-en-Laye, France) and 5'-RACE and 3'-RACE primers (Supplementary Table 1). The RACEPCR reactions were performed using the Advantage 2 PCR Enzyme System (Clontech-Takara Bio Europe, Saint-Germain-en-Laye, France). The obtained products were purified from agarose gel and cloned using the Strata Clone PCR Cloning Kit. Sequence data have been deposited at the GenBank database.

\section{Bioinformatic analysis}

The FastPCR v. 6.5.99 (http://primerdigital.com/fastp cr.html) and Universal ProbeLibrary Assay Design Center (http://www.roche-applied-science.com/sis/rtpcr/upl) were used for degenerated and qPCR primers designing, respectively. The identified sequence was analyzed using BLAST 2.2.25 (http://blast.ncbi.nlm.nih.gov/Blast.cgi), ExPASY (http://www.expasy.org/) and PRALINE (http:// www.ibi.vu.nl/programs/praline/). The ProtComp software was used to predict localization of the LIIDL protein with LocDB and PotLocDB databases (ProtComp v 8.0, http:// www.softberry.com). The dendrogram was constructed using the Phylogeny.fr (http://www.phylogeny.fr/simple_ phylogeny.cgii).

\section{Gene expression analyses}

The LIIDL expression was analyzed in different $\mathrm{AZ}$ variants: inactive AZ (Supplementary Fig. 1a), natural active AZ (Supplementary Fig. 1b), artificially activated AZ (Supplementary Fig. 1c, d) at 2, 4, 6, 8, 16 and $24 \mathrm{~h}$ time points of activation, as well as ABA, NDGA, ET or NBD treatments. We performed a Real-Time PCR (RT-qPCR) assay with a LightCycler 2.0 Carousel-Based System (ROCHE Diagnostics GmbH, Germany) and the LightCycler TaqMan Master Kit (ROCHE Diagnostics GmbH, Germany). The cDNA templates for RT-qPCR were prepared the same way as for the gene molecular cloning. The qPCR reactions were performed for the LIIDL (GenBank acc. no. KT716180), as well as for LlACT (GenBank acc. no. KP257588), which was used as a reference endogenous control for normalization purposes. The qPCR reactions with using gene-specific primers and UPL probes (Supplementary Table 1) were performed under the same conditions as described Frankowski et al. (2015b). Relative quantification was calculated using standard curves from serial dilutions of cDNA. The efficiency tested was $>99 \%$. Calculations and charts were prepared using LightCycler Real-Time PCR Systems (ROCHE Diagnostics GmbH, Germany) and SigmaPlot 2001 v.7.0. All data are the results of three separate samples with three replications of each cDNA and were presented as a mean \pm standard error (SE). Student's $t$ test was used to calculate the significant differences compared with the control.

\section{EPIP treatments}

In silico analysis of predicted LIIDL protein sequence revealed the presence of EPIP motif (HFSGFLPKRTHMPYSTPSRKHN) which was further synthesized by Novazym POLSKA s.c. (Certificate no. 170302-P013322, Supplementary Fig. 2). Synthetic EPIP solutions (1, 10 or $100 \mu \mathrm{M})$ prepared in $0.05 \%(\mathrm{v} / \mathrm{v})$ Tween 20 were applied by small brushes directly onto inactive flower AZs. Control AZs were treated with $0.05 \%$ Tween 20 solution only. The number of flowers per plant was calculated 4 days after each application. 


\section{Microscopy analysis}

Fresh fragments of inactive, active and activated AZs were fixed in mixture $4 \%$ paraformaldehyde (w/v, Fluka) and $0.2 \%(\mathrm{w} / \mathrm{v})$ glutaraldehyde (Sigma-Aldrich) in phosphatebuffered saline (PBS), pH 7.2 overnight at $4{ }^{\circ} \mathrm{C}$. Next material was dehydrated in increasing ethanol concentrations, supersaturated and then embedded in BMM or Unicryl resin (BBInternational, UK) at $-20{ }^{\circ} \mathrm{C}$ under UV light for polymerization. Semithin sections $(1 \mu \mathrm{m})$ were cut on an Ultracut microtome (Reichert-Jung, Germany) and were transferred on glass slides covered with Biobond (BBInternational, UK). Ultrathin sections (70 $\mathrm{nm}$ thick) were cut using the same type of microtome. For histological analyses, longitudinal sections were stained with $0.05 \%(\mathrm{w} / \mathrm{v})$ toluidine blue. The samples were observed in the LM Zeiss Axioplan microscope (Carl Zeiss, Germany). All images were obtained with a ProGres C3 digital camera using the ProGres CapturePro 2.6 software (Jenoptik AG, Germany).

For transmission electron microscopy ultrathin sections were mounted on formvar-coated nickel grids and stained for general contrast with $2 \%$ uranyl acetate followed by lead citrate. Observations were carried out using a Jeol TEM-1011 transmission electron microscope (JEOL, Japan).

\section{Results}

\section{Specific changes of $A Z$ cells accompanying $A Z$ activation}

Following abscission, AZ cells become easy to distinguish and were characterized by intensive divisions (Fig. 1a-d). The newly synthesized cell walls were formatted, as a consequence of anticlinal cell divisions approximately within 4-5 cell layers (Fig. 1c). Around vascular bundles, in the central area of flower pedicel, cells were totally different to the neighboring ones and were forming a strongly stained stripe indicating middle lamella dissolution and cell wall rupture (Fig. 1a, b). We observed big nuclei and heterogeneous cytoplasm enriched by many organelles and small vesicles (Fig. 1e). Figure 1f illustrates the occurrence of different
Fig. 1 The structure of naturally active flower abscission zone (AZ) in yellow lupine. AZs were collected from the pedicels fragments (1 $\mathrm{mm}$ above and $1 \mathrm{~mm}$ below flower bases) of fully opened naturally abscised flowers (Supplementary

Fig. 1b). For general observations, sections were stained with toluidine blue $(\mathbf{a}-\mathbf{d})$. The images of transmission electron microscopy (e-k) show magnifications of active $\mathrm{AZ}$ in different regions. Arrows denote cell plate forming between daughter cells $(\mathbf{c}, \mathbf{d}, \mathbf{g})$ and aggregation of nuclei (h). Cell organelle labeling: $c$ cytoplasm, $c h$ chloroplast, $c w$ cell wall, $n$ nuclei, nu nucleolus, $p$ plasmodesmata, $s$ starch grain, $v$ vacuole. Bars $=100 \mu \mathrm{M}(\mathbf{a}), 40 \mu \mathrm{M}(\mathbf{b}-\mathbf{d})$ and $10 \mu \mathrm{M}(\mathbf{e}-\mathbf{k})$
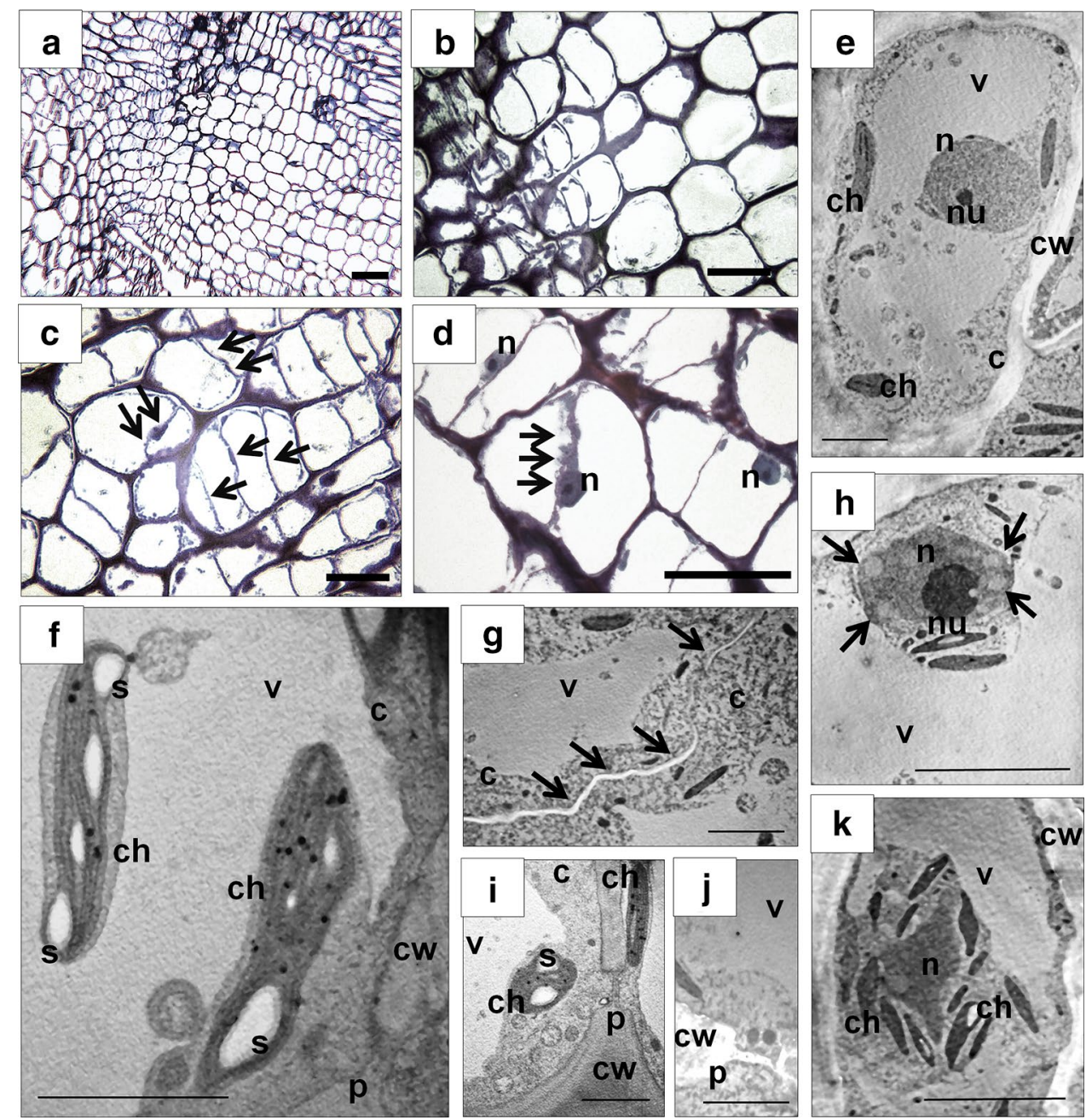
kinds of membrane vesicles associated with chloroplasts. The cell plate which was initially formed develops between the displaced daughter cells and a new cell wall creation was visible (Fig. 1g). The subsequent, mostly distinguishable, symptoms were characteristics branched plasmodesmata that interconnected adjacent cells (Fig. 1i, j). Typical active AZ cells had elongated-shaped chloroplasts, sometimes containing lipid-protein plastoglobuli and starch grains (Fig. 1f, i). Some of the nuclei were electron dense and condensed, with visible aggregates (Fig. 1h). Additionally, many of them became ameboid-shaped (Fig. 1k).

Cells of inactive AZ (Fig. 2) were totally distinct compared to naturally active AZ (Fig. 1) and were characterized by thick cell walls and almost homogenous cytoplasm (Fig. 2c, d). Small organelles in the peripheral regions of the cells were visible (Fig. 2c, e).

An artificial activation leads to changes at the cellular level (Fig. 3) similar to those presented in naturally active AZ cells (Fig. 1). After $2 \mathrm{~h}$ newly cell walls were formed between cells near the center of a vascular bundle (Fig. 3a). Subsequently, cytoplasm became more heterogeneous ( $4 \mathrm{~h}$ ) (Fig. 3b). Other symptoms were enlargement of nuclei and presence of many plasmodesmata (6 h) (Fig. 3c). Following activation round compartments in the vascular cells area $(8 \mathrm{~h})$ were detected (Fig. 3d). In the $16 \mathrm{~h}$ after flower removal, many small aggregates were diffused in the cytoplasm of the AZ cells (Fig. 3e). Changes visualized $24 \mathrm{~h}$ after abscission activation (Fig. 3f), were similar to those presented in naturally active AZ cells (Fig. 1).

\section{Isolation of LIIDL CDNA}

The LIIDL was obtained in RCR reactions with degenerated primers (Supplementary Fig. 3a) and 5'-,3'-RACEPCR (Supplementary Fig. 3b, c). The sequence of LIIDL cDNA (GenBank acc. no. KT716180) had a total length of 307 bp (Supplementary Fig. 4a). In silico analysis shown that the open reading frame of LIIDL cDNA encoded a 76 aa protein sequence (GenBank acc. no. AMH85930) with the predicted molecular mass of $8.91 \mathrm{kDa}$ and the isoelectric point of 11.64. The 22-amino acid EPIP domain occurs in LIIDL predicted protein sequence (Supplementary Fig. 4b). The EPIP composed of a variable region and a PIP motif containing conserved Pro residue (Supplementary Fig. 4a). What is more, LIIDL includes highly conserved KHN motif and secretory signal peptide at the
Fig. 2 Microscopy analysis of inactive flower abscission zone (AZ) (a-e) from yellow lupine. AZs were collected from the pedicels fragments ( $1 \mathrm{~mm}$ above and $1 \mathrm{~mm}$ below flower bases) of fully opened non-abscised flowers (Supplementary Fig. 1a). Enlarged region of $\mathrm{AZ}$ stained with toluidine blue is presented in (b). Transmission electron micrographs of inactive AZ (c-e). Cell organelle labeling: $c$ cytoplasm, $c h$ chloroplast, $c w$ cell wall, $v$ vacuole. Bars $=100 \mu \mathrm{M}(\mathbf{a}), 40 \mu \mathrm{M}(\mathbf{b})$ and $10 \mu \mathrm{M}(\mathbf{c}-\mathbf{e})$
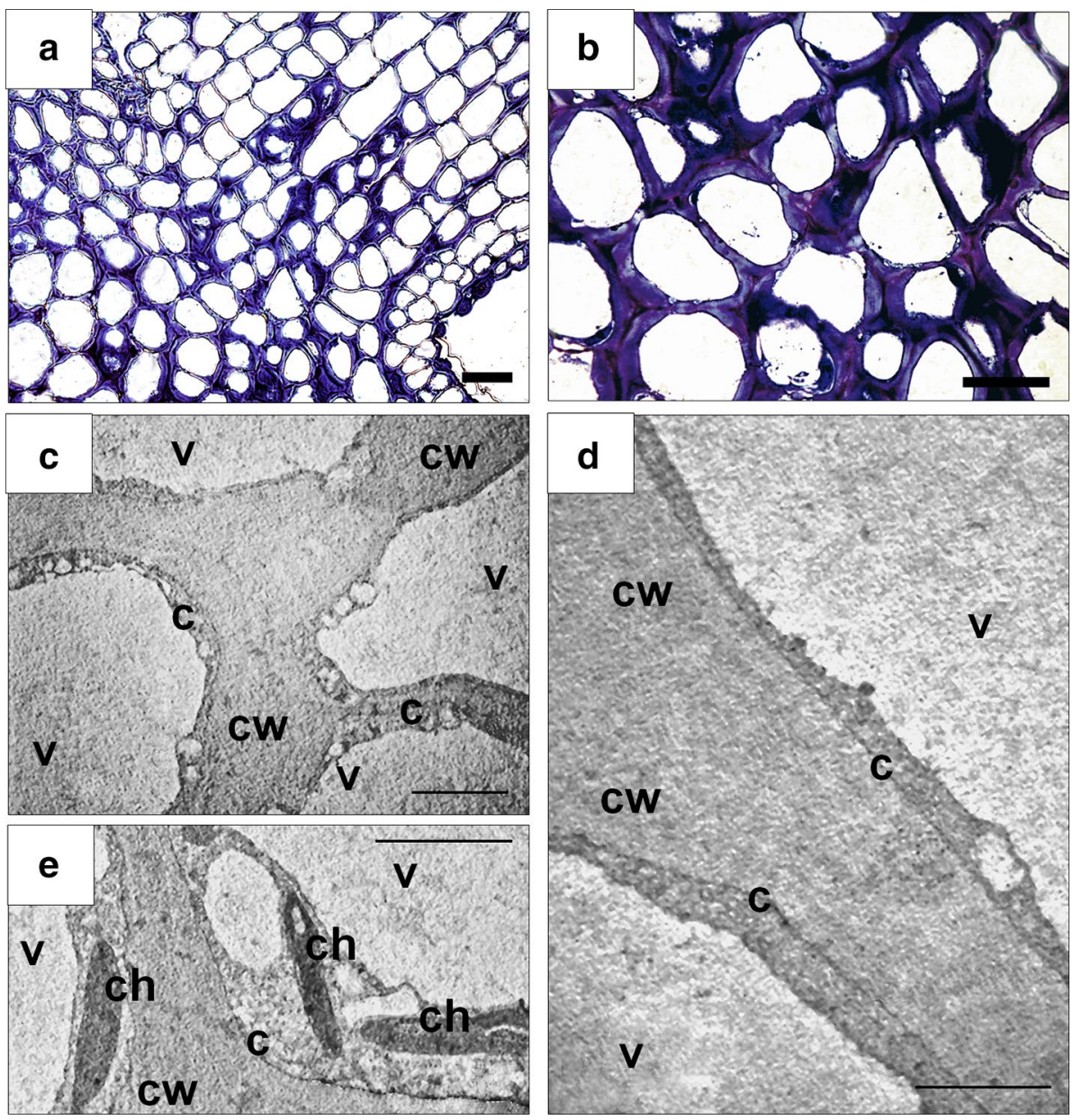
Fig. 3 Assessing the structural changes following artificial activation of flower abscission zone (AZ) in L. luteus L. Toluidine blue staining of $\mathrm{AZ}$ sections (excised $1 \mathrm{~mm}$ above and $1 \mathrm{~mm}$ below flower bases) collected 2, 4, 6, 8, 16 and $24 \mathrm{~h}$ after flower removal (Supplementary Fig. 1c, d). Bars $=40 \mu \mathrm{M}$
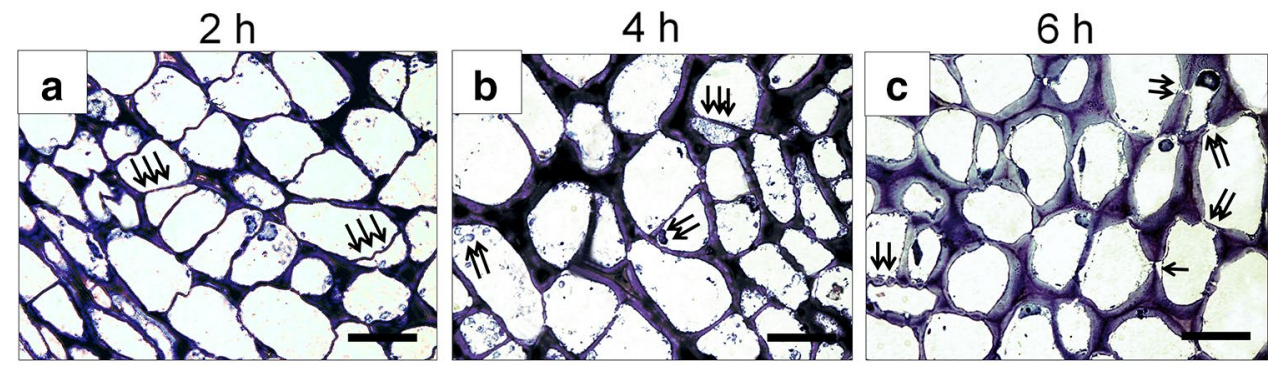

$8 \mathrm{~h}$

$16 \mathrm{~h}$
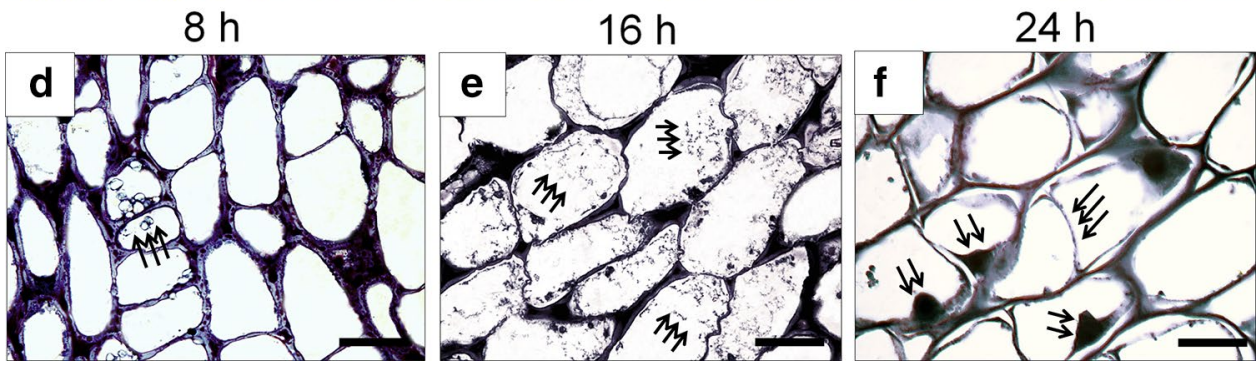

$\mathrm{N}$-terminus of protein (Supplementary Fig. 4a). A screen used ProtComp software revealed that predicted LIIDA is located in the extracellular space. In turn, the phylogenetic alignment between LIIDL amino acid sequence with other IDL members from different species shown that the predicted protein is closely related to IDA-like2 and IDAlike4 from G. max (Supplementary Fig. 5).

\section{EPIP peptide efficiently induces flower abscission}

We showed that exogenous synthetic EPIP peptide applied on flower AZs increased the number of abscised flowers as compared to control plants treated with $0.05 \%$ Tween 20 (Fig. 4). The observed effect was the strongest after application of $100 \mu \mathrm{M}$ solution ( 87\%). The $10 \mu \mathrm{M}$ EPIP peptide affected flower abortion less effectively, the noticed value was almost $10 \%$ lower than in control. EPIP peptide in $1 \mu \mathrm{M}$ solution did not affect flower abscission (Fig. 4).

\section{LIIDL transcriptional activity is induced following naturally and artificially stimulated flower abscission}

We observed that expression of $L I I D L$ was 70 -fold higher in in $\mathrm{AZ}$ cells prior to flower separation compared to control AZ (Fig. 5a). Interestingly, the LIIDL mRNA level gradually increased in flower AZ following its artificial activation by organ removal. A significant accumulation LIIDL transcript was observed starting from 6 to $24 \mathrm{~h}$ (Fig. 5b). It was above $40 \%$ higher when compared to inactive AZ. The maximum value was noticed in the last examined time variant $(24 \mathrm{~h})$.

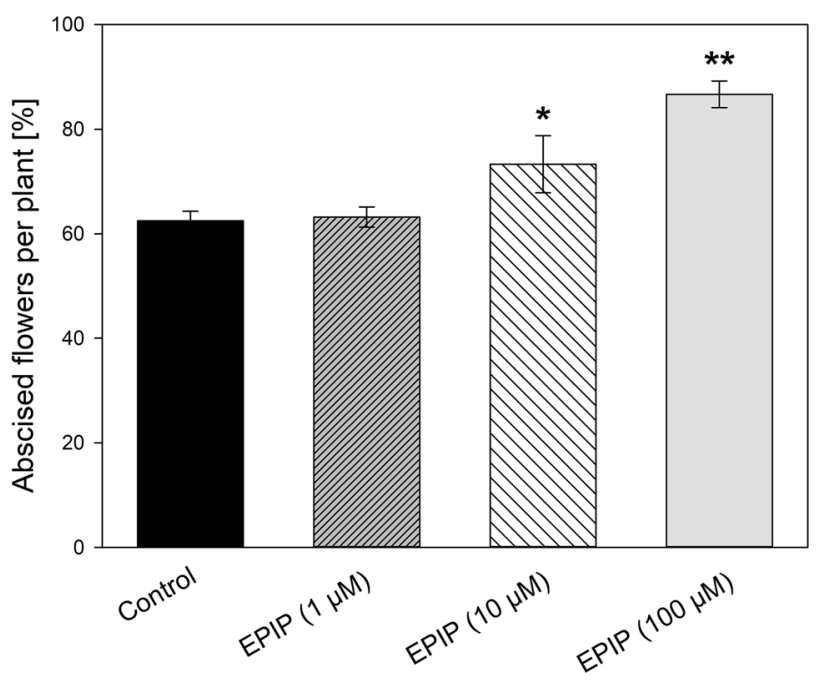

Fig. 4 The effect of EPIP synthetic peptide on flower abscission in yellow lupine. The 1,10 or $100 \mu \mathrm{M}$ solutions were applied on inactive flower abscission zones (AZs). Significant differences to plants treated with EPIP in comparison to control are indicated as $* * \mathrm{P}<0.01$ and $* \mathrm{P}<0.05$

\section{$A B A$ and ET are modulators of LIIDA expression in flower $A Z$}

The modifications of AZ cell structure $24 \mathrm{~h}$ after ABA or ET application (Fig. 6c, e) were similar to those, observed after its artificial activation (Fig. 3). Exogenous hormones caused an appearance of small organelles and visible granules (Fig. 6c, e). It should be pointed out that the effect of ET was stronger because stimulated also cellular divisions. The cellular structure after treatments with an inhibitor of ABA biosynthesis (NDGA) or ET action 


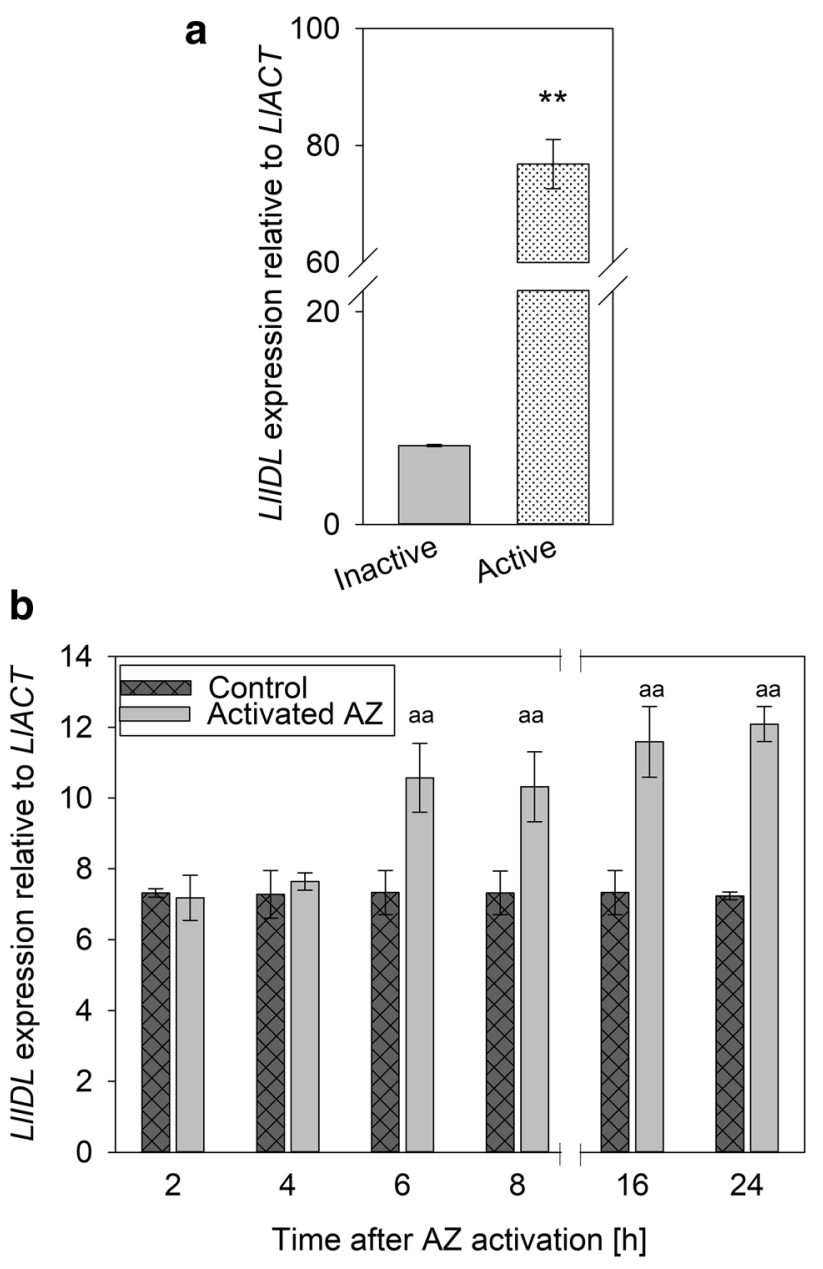

Fig. 5 Transcriptional activity of $L I I D L$ (related to LlACT) in natural active (a) and artificially activated (b) abscission zone (AZ) from $L$. luteus L. In parallel inactive AZs (control) were harvested. Significant differences to the natural active AZ in comparison to non-activated $\mathrm{AZ}$ are indicated as $* * \mathrm{P}<0.01$. Significant differences to artificially activated $\mathrm{AZ}$ in comparison to inactive $\mathrm{AZ}$ are indicated as ${ }^{\text {aa }} \mathrm{P}<0.01$

(NBD) is the confirmation of both hormone's action in AZ (Fig. 6d, f). In these cases, the cells were similar to those observed in inactive AZ (Fig. 2).

Exogenous ABA or ET triggered distinct changes in the LIIDL transcriptional activity (Fig. 6). Elevated expression occurred in 4 and $6 \mathrm{~h}$ after ABA treatment (Fig. 6a), whereas ET increased LIIDL expression after 8, 16 and $24 \mathrm{~h}$ (Fig. 6b). The positive effect of both hormones was reversed by NDGA or NBD (Fig. 6a, b). The inhibitor of ABA biosynthesis or ET action decreased mRNA content of the examined gene in 2 and $4 \mathrm{~h}$ after application. However, NDGA enhanced $L I I D L$ expression in the last time variant (24 h) (Fig. 6a).

\section{Discussion}

In the study of flower abscission, the model that has mainly been used is A. thaliana. The signaling pathway that controls abscission from ligand (IDA) to receptors (HSL2/HAE) has been proposed in this species (Butenko and Aalen 2012; Meng et al. 2016). However, little is still known about the coordination of this process in the significant economic crop, such as Lupinus luteus L., characterized by high seed protein content and the ability to nitrogen fixation. Insufficient yielding limited by excessive flower abscission is a major barrier to the expansion of lupine cultivation in Europe. Therefore, understanding of this process would help to regulate seed production. The place of flower detachment is an AZ, which is extremely sensitive to plant hormones. Nevertheless, the aspect of growth regulators in separation processes is not often examined, especially at the molecular level (Wilmowicz et al. 2016).

Changes occurred in natural active AZ, e.g. intensive divisions and newly synthesized cell wall (Fig. 1) may suggest a considerable activity of cells, but on the other hand, formed cells could initiate the processes of protective scar formation. This is in accordance with the reported cell proliferation in flower AZ of Euphorbia pulcherrima and Castanea fruit, however, it is not required for flower separation in Arabidopsis, tobacco, tomato, as well as Begonia (Lee et al. 2008; van Doorn and Stead 1997). Studies conducted on tissue and cell cultures indicate that divisions are part of the differentiation process that preceded the formation of new, functionally specialized certain types of cells (Schiavone and Racusen 1990). An occurrence of dense and heterogeneous cytoplasm and different kinds of membrane vesicles (Fig. 1e-g) may indicate vesicular trafficking, as well as induction and transport of crucial molecules involved in separation processes, such as cellwall hydrolyzing enzymes. For instance accumulation of cellulases, hydrolases, expansins or polygalacturonase, has been reported before in tomato, peach and Sambucus nigra (Belfield et al. 2005; Bonghi et al. 1992; Kalaitzis et al. 1997). Characteristic branched plasmodesmata in lupine AZ cells (Fig. 1i, j) confirmed extensive membrane trafficking. The plasmodesmata could provide communication and directional flow of regulatory molecules, e.g. RNA. These observations were also presented by Bar-Dror et al. (2011) in tomato. However, Scott et al. (1967) postulated that branched plasmodesmata in the vasculature of Phaseolus vulgaris significantly reduced levels of callose plugs during abscission.

Ultrastructural nuclei changes indicate DNA fragmentation and a possibility of programmed cell death occurring, like observed in tomato leaf AZ (Bar-Dror et al. 2011). 
Fig. 6 The impact of ABA and ET on the expression of LIIDL $(\mathbf{a}, \mathbf{b})$ and structural changes (c-f) in L. luteus L. floral abscission zones (AZs). Tissue fragments for qPCR experiments were collected 2, 4, 6, 8, 16 and $24 \mathrm{~h}$ after application of abscisic acid (ABA), or nordihydroguaiaretic acid (NDGA, an inhibitor of ABA biosynthesis) (a) and ethylene (ET) or 2,5-norbornadiene (NBD, ET action inhibitor) (b) treatments. The inactive $\mathrm{AZ}$ was treated with $0.05 \%$ Tween 20 solution (control). LlACT was used as a reference gene. Significant differences in LlIDL expression to the plants treated with $\mathrm{ABA}$ in comparison to Control are indicated as ${ }^{\text {aa }} \mathrm{P}<0.01$; to the plants treated with NDGA in comparison to Control are indicated as ${ }^{\mathrm{b}} \mathrm{P}<0.05$; to the plants treated with NDGA in comparison to ABA-treated plants are indicated as ${ }^{c c} \mathrm{P}<0.01$ and ${ }^{c} \mathrm{P}<$ 0.05 ; to the plants treated with ET in comparison to inactive $\mathrm{AZ}$ are indicated as ${ }^{\mathrm{dd}} \mathrm{P}<0.01$; to the plants treated with NBD in comparison to inactive $\mathrm{AZ}$ are indicated as ${ }^{\mathrm{e}} \mathrm{P}<0.05$; to the plants treated with NBD in comparison to ET-treated plants are indicated as ${ }^{\mathrm{ff}} \mathrm{P}<0.01$ and ${ }^{\mathrm{f}} \mathrm{P}<0.05$. Sections for toluidine blue staining were excised $24 \mathrm{~h}$ after each application $(\mathbf{c}-\mathbf{f})$
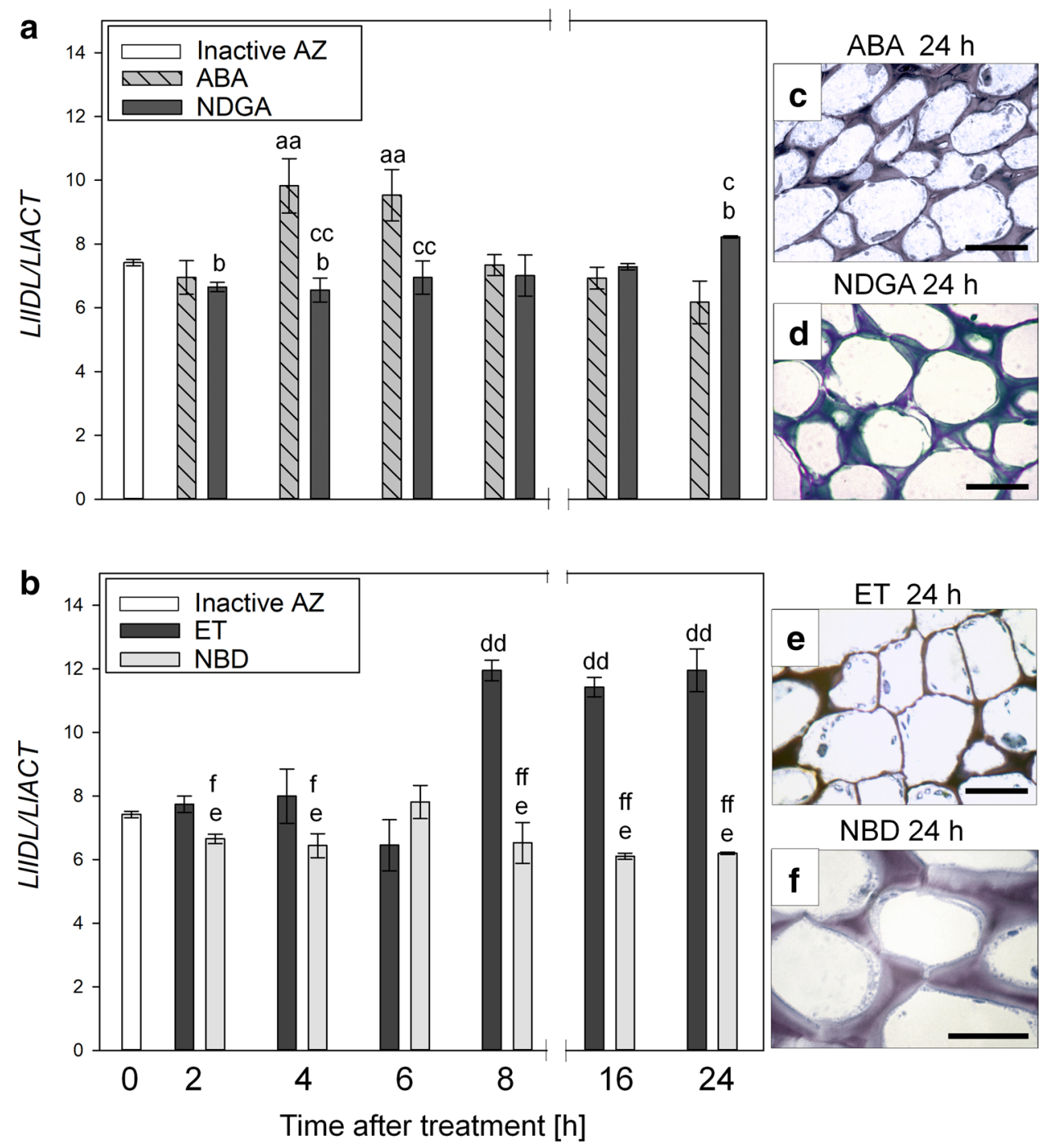

However, nuclei remained intact and there was no visible evidence of cell death after separation of oil palm fruit (Roongsattham et al. 2016). Another progressive cell degradation symptoms are the presence of oval-shaped chloroplasts containing large starch grains and thylakoidassociated plastoglobules (Fig. 1e, f, h, k). We suggest that starch is a source of energy for cell division, but also for the synthesis of new enzymes involved in the hydrolysis of macromolecules, which building cell compartments (Rolland et al. 2002). The phenomenon of plastoglobuli formation could be a part of photoprotection mechanism induced in oxidative stress conditions, as well as during chloroplasts senescing (Bréhélin et al. 2007).

To better examine the changes occur in early stages of flowers separation, we activated flower AZ by organ removal, as was previously described (Meir et al. 2010; Wilmowicz et al. 2016). The cellular divisions (Fig. 3a, b, d, f), formation of small aggregates (Fig. 3b, e), occurrence of many plasmodesmata (Fig. 3c), and the presence of big nuclei (Fig. 3c, f) may be explained again by high metabolic activity of these cells and synthesis and transport of crucial molecules involved in early separation processes, that ensure cellular communication within AZ (Taylor et al. 1991).

In our study, we isolated for the first time $I D L$ from $L$. luteus (LlIDL) (Supplementary Fig. 4a). The bioinformatic analysis of the nucleotide sequence revealed that predicted LIIDL contains motifs and domains characteristic for other plants IDA/IDL (Supplementary Fig. 4a), among them $\mathrm{N}$-terminal secretory signal peptide and EPIP motif located at the $\mathrm{C}$-terminal. What is more, an analysis performed with using ProtComp confirmed extracellular space localization of LIIDA, which may suggest that, similarly like other IDA/ IDL, N-terminal motif enables proper translocation of this protein to ensure interactions with the receptor (Stenvik et al. 2008). Aalen (2013) and Butenko et al. (2003) postulated that secreted IDA/IDL move within apoplast and is involved in cell-to-cell communication. Interestingly, LcIDL1 from $L$. chinensis has detected also in the cytoplasm area, suggesting 
that mechanism of IDA releasing is controlled by other factors (Ying et al. 2016). Predicted LIIDL contains conserved 22-amino acid EPIP domain composed of the variable region and a PIP motif (Supplementary Fig. 4b). PIP motif contains Pro residue putatively post-transcriptionally modified to hydroxyproline that is crucial for interactions of IDA with HSL2/HAE receptors. Additionally KHN motif responsible for co-receptor recognition site was localized within PIP motif (Butenko et al. 2003; Santiago et al. 2016).

It was confirmed that EPIP motif determines IDA/IDL activity (Santiago et al. 2016; Stenvik et al. 2008), therefore we synthesized EPIP on the basis of LIIDL and performed physiological experiments in order to define the concentration of EPIP solution, which induces a response. Synthetic EPIP peptide applied directly on flower AZs strongly stimulated abortion of these organs (Fig. 4). What is more, positive correlation between observed physiological effect and solution concentration occured. To the best of our knowledge, this is the first report concerning analyses performed in vivo on plants growing under phytotron conditions. However, in vitro studies revealed that synthetic EPIP peptides rescued ida mutant and induced early floral abscission in $A$. thaliana wild type (Stenvik et al. 2008). Overall, we concluded that EPIP motif is sufficient to induce $\mathrm{AZ}$ activation and consequently flowers separation. Consistent with these findings, we designed specific primers and probe on the basis of $L I I D L$ nucleotide fragment encoding exactly putative EPIP motif and we performed quantification of gene expression. Transcriptional activity of LIIDL was significantly higher in naturally active flower AZ than in control (Fig. 5a), confirming LIIDA involvement in separation processes. In A. thaliana IDA specifically expressed in floral AZ and mutant analysis revealed that is essential for abscission (Butenko et al. 2003). Additionally, more recently, Ying et al. (2016) reported increasing LcLIDL1 expression in flower AZ following this organ development. In L. luteus cumulative changes observed in time at the cellular level after artificial flower AZ activation were correlated with gradual accumulation of $L I I D L$ transcript (Fig. 5b). It supports the thesis about $L I I D L$ contribution in the early steps of $\mathrm{AZ}$ activation, which is a novel report in this area.

Phytohormones are signal molecules produced within the plant, critical in modulating many physiological responses. Our previous works clearly demonstrated that ABA and ET are strong stimulators of flower abortion in L. luteus (Frankowski et al. 2017; Wilmowicz et al. 2016). The presented paper provides new information-both hormones cause modifications of cell structure in AZ (Fig. 6c-f), like these observed after its artificial activation (Fig. 3a-f). It suggests that $\mathrm{ABA}$ and ET may turn on abscission processes. In view of these results, we put forward a hypothesis that both hormones can regulate the transcriptional activity of potential abscission activator-LIIDL. Interestingly, application of ET stimulated LlIDL mRNA level in the last three time variants, while ABA generated this effect in the first hours after application (Fig. 6a, b). These results are consistent with our previous investigations. We have found that expression of ET biosynthesis genes (LlACS and $L l A C O$ ) increased started from $8 \mathrm{~h}$ after artificial AZ activation (Frankowski et al. 2017). Tucker and Yang (2012) showed that ET treatment caused an accumulation of 12 soybean IDAs mRNAs, even $72 \mathrm{~h}$ after treatment. Similar results were obtained by Ying et al. (2016) in litchi. We have demonstrated for the first time how ABA influences LIIDL expression. Our findings suggest that sensitivity of LIIDA on hormonal factors is different. The response for ET is later than ABA, although is more effective, so these phytohormones can act in various stages during AZ activation process, as we previously demonstrated (Wilmowicz et al. 2016).

Collectively, this study provides promising advances in our understanding of separation processes in yellow lupine and the impact of LIIDA on the early steps of floral AZ activation-the mechanism that involves ABA and ET. Our data provide crucial information for the elucidation of new potential molecular markers of abscission contributing to the advance in knowledge that will consequently improve the yield of a great economic potential plant.

Acknowledgements The authors would like to thank Dr. Krzysztof Zienkiewicz for his help with transmission electron microscopy. This work was supported by the Polish Ministry of Agriculture and Rural Development [Grants Nos. 149/2011 and 222/2015] and funds provided by the consortium of Agrifood International Doctorate School and Agrifood Campus of International Excellence (eidA3-ceiA3) funding throughout the program for Ph.D. co-supervision for foreign students (Agata Kućko).

Open Access This article is distributed under the terms of the Creative Commons Attribution 4.0 International License (http://creativeco mmons.org/licenses/by/4.0/), which permits unrestricted use, distribution, and reproduction in any medium, provided you give appropriate credit to the original author(s) and the source, provide a link to the Creative Commons license, and indicate if changes were made.

\section{References}

Aalen RB (2013) Maturing peptides open for communication. J Exp Bot 64:5231-5235

Agustí J, Zapater M, Iglesias DJ, Cercós M, Tadeo FR, Talón M (2007) Differential expression of putative 9-cis-epoxycarotenoid dioxygenases and abscisic acid accumulation in water stressed vegetative and reproductive tissues of citrus. Plant Sci 172:85-94

Aloni B, Kami L, Zaidman Z, Schaffer AA (1996) Changes of carbohydrates in pepper (Capsicum annиum L.) flowers in relation to their abscission under different shading regimes. Ann Bot 78:163-168

Bar-Dror T, Dermastia M, Kladnik A, Znidaric MT, Novak MP, Meir S, Burd S, Philosoph-Hadas S, Ori N, Sonego L, Dickman MB, Lers 
A (2011) Programmed cell death occurs asymmetrically during abscission in tomato. Plant Cell 23:4146-4163

Belfield EJ, Ruperti B, Roberts JA, McQueen-Mason S (2005) Changes in expansin activity and gene expression during ethylene-promoted leaflet abscission in Sambucus nigra. J Exp Bot 56:817-823

Bonghi C, Rascio N, Ramina A, Casadoro G (1992) Cellulase and polygalacturonase involvement in the abscission of leaf and fruit explants of peach. Plant Mol Biol 20:839-848

Botton A, Eccher G, Forcato C, Ferrarini A, Begheldo M, Zermiani M, Moscatello S, Battistelli A, Velasco R, Ruperti B, Ramina A (2011) Signaling pathways mediating the induction of apple fruitlet abscission. Plant Physiol 155:185-208

Bréhélin C, Kessler F, van Wijk KJ (2007) Plastoglobules: versatile lipoprotein particles in plastids. Trends Plant Sci 12:260-266

Burr CA, Leslie ME, Orlowski SK, Chen I, Wright CE, Daniels MJ, Liljegren SJ (2011) CAST AWAY, a membrane-associated receptor-like kinase, inhibits organ abscission in Arabidopsis. Plant Physiol 156:1837-1850

Butenko MA, Aalen RB (2012) Receptor ligands in development. In: Tax F, Kemmerling B (eds) Receptor-like kinases in plants: from development to defense. Springer-Verlag, Berlin, Heidelberg, pp 195-226

Butenko MA, Patterson SE, Grini PE, Stenvik GE, Amundsen SS, Mandal A, Aalen RB (2003) INFLORESCENCE DEFICIENT IN ABSCISSION controls floral organ abscission in Arabidopsis and identifies a novel family of putative ligands in plants. Plant Cell 15:2296-2307

Cho SK, Larue CT, Chevalier D, Wang H, Jinn T-L, Zhang S, Walker JC (2008) Regulation of floral organ abscission in Arabidopsis thaliana. Proc Natl Acad Sci USA 105:15629-15634

Couzigou JM, Magne K, Mondy S, Cosson V, Clements J, Ratet P (2016) The legume NOOT-BOP-COCH-LIKE genes are conserved regulators of abscission, a major agronomical trait in cultivated crops. New Phytol 209:228-240

Estornell LH, Agusti J, Merelo P, Talon M, Tadeo FR (2013) Elucidating mechanisms underlying organ abscission. Plant Sci 199:48-60

Estornell LH, Wildhagen M, Pérez-Amador MA, Talón M, Tadeo FR, Butenko MA (2015) The IDA peptide controls abscission in Arabidopsis and citrus. Front Plant Sci 6:1003

Frankowski K, Kućko A, Zienkiewicz A, Zienkiewicz K, Alché JD, Kopcewicz J, Wilmowicz E (2015a) Profiling the BLADE-ONPETIOLE gene expression in the abscission zone of generative organs in Lupinus luteus. Acta Physiol Plant 37:220

Frankowski K, Wilmowicz E, Kućko A, Zienkiewicz A, Zienkiewicz $\mathrm{K}$, Kopcewicz J (2015b) Molecular cloning of the BLADE-ON$P E T I O L E$ gene and expression analyses during nodule development in Lupinus luteus. J Plant Physiol 179:35-39

Frankowski K, Kućko A, Zienkiewicz A, Zienkiewicz K, Kopcewicz J, de Dios JD, Wilmowicz E (2017) Ethylene-dependent effects on generative organ abscission of Lupinus luteus. Acta Soc Bot Pol 86:3540

Gomez-Jimenez MC, Paredes MA, Gallardo M, Sanchez-Calle IM (2010) Mature fruit abscission is associated with up-regulation of polyamine metabolism in the olive abscission zone. J Plant Physiol 167:1432-1441

Kalaitzis P, Solomos T, Tucker ML (1997) Three different polygalacturonases are expressed in tomato leaf and flower abscission, each with a different temporal expression pattern. Plant Physiol 113:1303-1308

Lee Y, Derbyshire P, Knox JP, Hvoslef-Eide AK (2008) Sequential cell wall transformations in response to the induction of a pedicel abscission event in Euphorbia pulcherrima (poinsettia). Plant $\mathrm{J}$ 54:993-1003

Liu B, Butenko MA, Shi CL, Bolivar JL, Winge P, Stenvik GE, Vie AK, Leslie ME, Brembu T, Kristiansen W, Bones AM, Patterson
SE, Liljegren SJ, Aalen RB (2013) NEVERSHED and INFLORESCENCE DEFICIENT IN ABSCISSION are differentially required for cell expansion and cell separation during floral organ abscission in Arabidopsis thaliana. J Exp Bot 64:5345-5357

Meir S, Philosoph-Hadas S, Sundaresan S, Selvaraj KSV, Burd S, Ophir R, Kochanek B, Reid MS, Jiang CZ, Lers A (2010) Microarray analysis of the abscission-related transcriptome in the tomato flower abscission zone in response to auxin depletion. Plant Physiol 154:1929-1956

Meng X, Zhou J, Tang J, Li B, de Oliveira MVV, Chai J, He P, Shan L (2016) Ligand-induced receptor-like kinase complex regulates floral organ abscission in Arabidopsis. Cell Rep 14:1330-1338

Prusiński J, Borowska M (2001) Degree of success of legume cultivars registered by the center for cultivar testing over the period of market economy. Acta Scientiarum Polonorum Agricultura 6:3-16

Rolland F, Moore B, Sheen J (2002) Sugar sensing and signaling in plants. Plant Cell 14:185-205

Roongsattham P, Morcillo F, Fooyontphanich K, Jantasuriyarat C, Tragoonrung $S$ (2016) Cellular and pectin dynamics during abscission zone development and ripe fruit abscission of the monocot oil palm. Front Plant Sci 26:507-540

Santiago J, Brandt B, Wildhagen M, Hohmann U, Hothorn LA, Butenko MA, Hothorn M (2016) Mechanistic insight into a peptide hormone signaling complex mediating floral organ abscission. eLife 8:e15075

Schiavone FM, Racusen RH (1990) Microsurgery reveals regional capabilities for pattern reestablishment in somatic carrot embryos. Dev Biol 141:211-219

Scott PC, Miller LW, Webster BD, Leopold AC (1967) Structural changes during bean leaf abscission. Am J Bot 54:730-734

Shi CL, Stenvik GE, Vie AK, Bones AM, Pautot V, Proveniers M, Aalen RB, Butenko MA (2011) Arabidopsis class I KNOTTEDlike homeobox proteins act downstream in the IDA-HAE/HSL2 floral abscission signaling pathway. Plant Cell 23:2553-2567

Stenvik GE, Butenko MA, Urbanowicz BR, Rose JK, Aalen RB (2006) Overexpression of INFLORESCENCE DEFICIENT IN ABSCIS$S I O N$ activates cell separation in vestigial abscission zones in Arabidopsis. Plant Cell 18:1467-1476

Stenvik GE, Tandstad NM, Guo Y, Shi CL, Kristiansen W, Holmgren A, Clark SE, Aalen RB, Butenko MA (2008) The EPIP peptide of INFLORESCENCE DEFICIENT IN ABSCISSION is sufficient to induce abscission in Arabidopsis through the receptor-like kinases HAESA and HAESA-LIKE2. Plant Cell 20:1805-1817

Taylor JE, Tucker GA, Lasslett Y, Smith CJS, Arnold CM, Watson CF, Schuch W, Grierson D, Roberts JA (1991) Polygalacturonase expression during leaf abscission of normal and transgenic tomato plants. Planta 183:133-138

Tucker ML, Yang R (2012) IDA-like gene expression in soybean and tomato leaf abscission and requirement for a diffusible stellar abscission signal. AoB Plants. https://doi.org/10.1093/aobpla/ pls035

van Doorn WG, Stead A (1997) Abscission of flowers and floral parts. J Exp Bot 48:821-837

Wilmowicz E, Frankowski K, Kućko A, Świdziński M, de Alché JD, Nowakowska A, Kopcewicz J (2016) The influence of abscisic acid on the ethylene biosynthesis pathway in the functioning of the flower abscission zone in Lupinus luteus. J Plant Physiol 206:49-58

Ying P, Li C, Liu X, Xia R, Zhao M, Li J (2016) Identification and molecular characterization of an IDA-like gene from litchi, LcIDL1, whose ectopic expression promotes floral organ abscission in Arabidopsis. Sci Rep 6:37135 